\title{
Análise conjunta de indicadores na viabilidade econômica do confinamento de novilhos recebendo dietas com diferentes níveis de concentrado
}

\author{
[Joint analysis of economic viability indicators of feedlot steers fed diets with \\ different concentrate levels] \\ J.R.P. Rosa, P.S. Pacheco, E.A. Fabricio, A. Camera, G.S. Maysonnave, G.I.O. Machado
}

Universidade Federal de Santa Maria - Campus Santa Maria, RS

RESUMO

O objetivo deste estudo foi avaliar a viabilidade econômica, por meio da análise conjunta de indicadores financeiros expressos por animal, da terminação em confinamento de novilhos Aberdeen Angus recebendo dietas com diferentes níveis de concentrado (NC): 25, 40, 55 ou $70 \%$ (base na matéria seca). Foram consideradas cotações históricas consecutivas dos anos de 2003 a 2014. A análise univariada caracterizou-se por delineamento inteiramente ao acaso, com quatro tratamentos e quatro repetições, procedendo-se à análise de regressão. A análise multivariada consistiu de agrupamento (cluster). Pela análise univariada, houve similaridade para os indicadores financeiros, com médias para margem bruta de R \$ 173,21; margem líquida de $\mathrm{R} \$ 163,73$; lucro de $\mathrm{R} \$ 110,61$; valor presente líquido de $\mathrm{R} \$ 93,31$; índice benefício: custo de 1,048; retorno adicional sobre o investimento de 1,17\% a.m.; taxa interna de retorno de 2,04\% a.m. e payback descontado de 1,36 mês. Pela análise de cluster, o NC de 55\% apresentou maior discrepância em relação aos demais níveis, enquanto 40 e $70 \%$ foram os mais próximos. A análise conjunta dos indicadores financeiros indicou viabilidade do confinamento de novilhos, independentemente do nível de concentrado.

Palavras-chave: análise de investimentos, análise de agrupamento, eficiência econômica, projetos de investimento, tomada de decisão

\begin{abstract}
The objective of this study was to evaluate the economic viability, through the joint analysis of financial indicators expressed by animal, of the Aberdeen Angus steers feedlot finished fed diets with different levels of concentrate (CL): 25, 40, 55 or 70\% (on dry matter basis). Consecutive historical quotes of years 2003 to 2014 were considered. Univariate analysis was characterized by a completely randomized design with four treatments and four replications, proceeding regression analysis. Multivariate analysis consisted of grouping (cluster). The univariate analysis showed similarity to the financial indicators with averages to gross margin of $R \$ 173.21$; net margin of $R \$ 163.73$; income of $R \$ 110.61$; net present value of $R \$ 93.31$; benefit: cost ratio of 1.048; additional return on investment of $1.17 \%$ per month; internal rate of return of $2.04 \%$ per month and discounted payback of 1.36 months. By cluster analysis, 55\% CL presented greater discrepancy in relation to other levels, while 40 and $70 \%$ were the nearest. The analysis of the financial indicators indicated feasibility of feedlot steers, regardless of the concentrate level.
\end{abstract}

Keywords: decision-making, economic efficiency, grouping analysis, investment analysis, investment projects

\section{INTRODUÇÃO}

Além do incremento no uso do confinamento como sistema de terminação de bovinos no Brasil, na ordem de $105 \%$ nos últimos 10 anos (2006 a 2015 - Anuário..., 2015), tem-se verificado uso de níveis elevados de concentrado

Recebido em 20 de julho de 2016

Aceito em 9 de setembro de 2016

E-mail: joilmaro@yahoo.com.br na dieta dos animais (Millen et al., 2009; Costa Júnior et al., 2013).

A maioria dos estudos sobre níveis de concentrado abordam aspectos relacionados com o desempenho zootécnico, como ganho de peso, consumo e conversão alimentar. No entanto, tem-se verificado, por parte de técnicos, produtores e consultores, a necessidade de informações complementares sobre a viabilidade 
econômica do confinamento para terminação de bovinos de corte, quando se incrementa o uso de concentrado na dieta.

Nesse sentido, a tomada de decisão apresenta complexidade cada vez maior, e na etapa da viabilidade econômica, Souza e Clemente (2009) sugerem a análise conjunta de indicadores financeiros, visando quantificar, de maneira determinística, aspectos relacionados com o retorno e também com o risco do investimento.

Seguindo essa metodologia, Pacheco et al. (2012) e Pacheco et al. (2014a) demonstraram a relevância da estimativa e da interpretação conjunta de indicadores financeiros na análise econômica da terminação de novilhos em confinamento. No entanto, essa abordagem metodológica necessita de maior difusão, tendo em vista sua relevância gerencial.

O objetivo deste estudo foi avaliar a viabilidade econômica do desempenho em confinamento de novilhos Aberdeen Angus recebendo dietas com diferentes níveis de concentrado, por meio da avaliação conjunta de indicadores financeiros.

\section{MATERIAL E MÉTODOS}

O experimento foi conduzido no Departamento de Zootecnia da Universidade Federal de Santa Maria, estado do Rio Grande do Sul, Brasil.

Foram utilizados 16 novilhos Aberdeen Angus, contemporâneos e provenientes do mesmo rebanho, com idade média de 16 meses. Na fase de recria, os animais foram mantidos em pastagem de capim-arroz (Echinochloa colonum) e capim-elefante (Penisetum purpureum), recebendo sal mineral.

Foram avaliados quatro níveis de concentrado na dieta, com base na matéria seca: $25,40,55$ e $70 \%$.

Detalhes do manejo na fase de terminação em confinamento (após 30 dias de adaptação) constam em Rosa (2006), em cujo estudo os animais foram confinados em baias individuais cobertas, com piso e bebedouros de concreto e comedouro individual de madeira. $\mathrm{O}$ fornecimento da dieta ocorreu às oito e às 14 horas, à vontade, procurando manter sobra de $10 \%$ da quantidade ofertada. Estabeleceu-se o peso de abate de $450 \mathrm{~kg}$ para todos os tratamentos, sendo a média obtida de $452 \mathrm{~kg}$.
A dieta dos animais foi ajustada em teor de proteína bruta $(\mathrm{PB})$ para todos os tratamentos (isoproteicas $=13,35 \% \mathrm{~PB}$ ), pressupondo consumo médio diário de $2,5 \%$ do peso vivo e ganho de peso médio diário de $1,4 \mathrm{~kg} / \mathrm{animal}$ (Nutrient..., 1996). O volumoso utilizado foi a silagem do híbrido de milho (Zea mays, L.) AG5011 com 38,5\% de matéria seca e 7,3\% de proteína bruta. As proporções dos ingredientes utilizados para os níveis de concentrado constam na Tab. 1.

Tabela 1. Composição do concentrado, expresso em matéria seca (MS), de acordo com o nível de concentrado

\begin{tabular}{lcccc}
\hline $\begin{array}{l}\text { Ingredientes do } \\
\text { concentrado }\end{array}$ & \multicolumn{4}{c}{ Nível de concentrado, \% MS } \\
\cline { 2 - 5 } & \multicolumn{1}{c}{45} & \multicolumn{1}{c}{40} & \multicolumn{1}{c}{55} \\
\hline Grão de milho & 20,00 & 40,00 & 49,60 & 55,00 \\
moído, kg & & & & \\
Farelo de trigo, kg & 10,00 & 20,00 & 24,80 & 27,50 \\
Farelo de soja, kg & 66,40 & 37,10 & 23,45 & 15,71 \\
Minerais, kg & 3,6 & 2,90 & 2,15 & 1,79 \\
Ionóforo - & 75,00 & 46,90 & 34,10 & 26,80 \\
Rumensin®, g & & & & \\
\hline
\end{tabular}

Para avaliação econômica, foram utilizadas metodologias descritas em Pacheco et al. (2014b). Considerou-se cada proporção de concentrado na dieta como projetos de investimento mutuamente excludentes. $\mathrm{O}$ método determinístico foi empregado, e foram assumidos valores fixos (conhecidos) para os itens que compõem os custos bem como os indicadores de retorno e risco, tomando-se como base cotações históricas de 12 anos consecutivos (2003 a 2014) para a região Sul do Brasil, obtidos pelas fontes: IEA - Instituto de Economia Agrícola de São Paulo - e Anualpec - Anuário Brasileiro da Pecuária.

$\mathrm{Na}$ Tab. 2, constam os valores dos coeficientes utilizados nas estimativas dos indicadores financeiros.

Na Tab. 3, estão apresentadas as características de desempenho dos animais (adaptado de Rosa, 2006), utilizadas na estimativa dos custos e dos indicadores financeiros.

Os custos estimados/kg MS da dieta com o incremento no nível de concentrado constam na Tab. 4. 
Tabela 2. Coeficientes utilizados nas estimativas dos indicadores financeiros da terminação em confinamento de novilhos recebendo diferentes proporções de concentrado na dieta

\begin{tabular}{|c|c|c|c|c|c|}
\hline \multirow{2}{*}{ Itens } & \multirow{2}{*}{ Unidade } & \multicolumn{4}{|c|}{ Nível de concentrado, \% MS } \\
\hline & & 25 & 40 & 55 & 70 \\
\hline Número de períodos & meses & 4 & 4 & 4 & 4 \\
\hline Taxa mínima de atratividade $-\mathrm{TMA}^{1}$ & $\%$ a.m. & 0,8667 & 0,8667 & 0,8667 & 0,8667 \\
\hline $\begin{array}{l}\text { Instalações, máquinas, implementos, } \\
\text { equipamentos }{ }^{2}\end{array}$ & $\mathrm{R} \$$ /animal & 709,00 & 709,00 & 709,00 & 709,00 \\
\hline Vida útil das instalações e equipamentos & anos & 25 & 25 & 25 & 25 \\
\hline Vida útil das máquinas e implementos & anos & 10 & 10 & 10 & 10 \\
\hline Boi magro 3 & $\mathrm{R} \$$ a prazo $/ \mathrm{kg}$ vivo & 3,19 & 3,19 & 3,19 & 3,19 \\
\hline Boi gordo ${ }^{3}$ & $\mathrm{R} \$$ a prazo $/ \mathrm{kg}$ vivo & 3,60 & 3,60 & 3,60 & 3,60 \\
\hline Boi gordo ${ }^{3}$ & R\$ a prazo/@ & 108,00 & 108,00 & 108,00 & 108,00 \\
\hline Salário mínimo 3 & $\mathrm{R} \$ / \mathrm{mês}$ & 592,00 & 592,00 & 592,00 & 592,00 \\
\hline Valor da terra ${ }^{3}$ & $\mathrm{R} \$ / \mathrm{ha}$ & $9,370,00$ & $9,370,00$ & $9,370,00$ & $9,370,00$ \\
\hline Volumoso $^{4}$ & $\mathrm{R} \$ / \mathrm{kg} \mathrm{MS}$ & 0,32 & 0,32 & 0,32 & 0,32 \\
\hline Concentrado $^{4}$ & $\mathrm{R} \$ / \mathrm{kg} \mathrm{MS}$ & 1,09 & 0,86 & 0,75 & 0,69 \\
\hline Mão de obra contratada/diarista ${ }^{5}$ & $\mathrm{R} \$$ /animal & 9,96 & 8,98 & 8,98 & 7,67 \\
\hline Assistência técnica ${ }^{6}$ & $\begin{array}{l}\text { Salários } \\
\text { mínimos/mês }\end{array}$ & 2 & 2 & 2 & 2 \\
\hline
\end{tabular}

${ }^{1}$ TMA $(\%$ a.m. $)=(1+10,91 \% \text { a.a. })^{1 / 12}-1$.

${ }^{2}$ Considerou-se a capacidade estática das instalações de 1.000 bois.

${ }^{3}$ Obtido de Anuário... (2015).

${ }^{4}$ Alguns itens de custos obtidos de Anuário... (2015).

${ }^{5}$ Considerou-se 1 homem dia/500 bois confinados.

${ }^{6}$ Valor para 1.000 bois, considerando-se duas visitas mensais à propriedade. $\mathrm{R} \$=0,40$ US $\$$.

Tabela 3. Características de desempenho dos animais de acordo com o nível de concentrado na dieta

\begin{tabular}{lcccccrrr}
\hline \multicolumn{1}{c}{ Itens } & \multicolumn{3}{c}{ Nível de concentrado, \% MS } & Média & $\mathrm{CV}, \%$ & P $>\mathrm{F}$ \\
\cline { 2 - 5 } & 25 & 40 & 55 & 70 & & & & \\
\hline Tempo de alimentação & 112 & 110 & 100 & 84 & 101 & - & - \\
Peso inicial, kg & 325 & 320 & 315 & 322 & 321 & 6,00 & 0,9294 \\
Peso final, kg & 461 & 449 & 442 & 456 & 452 & 4,00 & 0,6000 \\
Ganho de peso total, kg & 136 & 129 & 127 & 134 & 132 & 7,87 & 0,6833 \\
Consumo matéria seca total, $\mathrm{kg}^{1}$ & 982,24 & $1.061,50$ & 976,00 & 890,68 & 984,05 & 5,51 & 0,0176 \\
\hline
\end{tabular}

${ }^{1} \hat{\mathrm{Y}}_{\mathrm{i}}=719,15+15,66 \mathrm{NC}_{\mathrm{i}}-0,19 \mathrm{NC}_{\mathrm{i}}{ }_{\mathrm{i}} ; \mathrm{R}^{2}=0,61$; ponto de máxima $=41,2 \%$ concentrado.

Tabela 4. Demonstração do custo da dieta experimental de acordo com a composição

\begin{tabular}{|c|c|c|c|c|}
\hline Ingredientes & $\begin{array}{c}\text { Composição } \\
\text { da dieta, \% MS } \\
\text { (a) }\end{array}$ & $\begin{array}{c}\mathrm{R} \$ / \mathrm{kg} \mathrm{MS} \\
\text { (b) } \\
\end{array}$ & $\begin{array}{c}\mathrm{R} \$ / \mathrm{kg} \text { MS da } \\
\operatorname{dieta}^{1}\end{array}$ & $\%$ do tota \\
\hline Volumoso & 75,0 & 0,32 & 0,24 & 46,6 \\
\hline Concentrado & 25,0 & 1,10 & 0,28 & 53,4 \\
\hline Total (volumoso + concentrado) & 100,0 & - & 0,52 & 100,0 \\
\hline Volumoso & 60,0 & 0,32 & 0,19 & 35,8 \\
\hline Concentrado & 40,0 & 0,86 & 0,34 & 64,2 \\
\hline Total (volumoso + concentrado) & 100,0 & - & 0,54 & 100,0 \\
\hline Volumoso & 45,0 & 0,32 & 0,14 & 25,9 \\
\hline Concentrado & 55,0 & 0,75 & 0,41 & 74,1 \\
\hline Total (volumoso + concentrado) & 100,0 & - & 0,56 & 100,0 \\
\hline Volumoso & 30,0 & 0,32 & 0,10 & 16,6 \\
\hline Concentrado & 70,0 & 0,69 & 0,48 & 83,4 \\
\hline Total (volumoso + concentrado) & 100,0 & - & 0,58 & 100,0 \\
\hline
\end{tabular}

${ }^{1}$ Dieta refere-se a volumoso + concentrado: $b^{*}(\mathrm{a} / 100) . \mathrm{R} \$=0,40$ US $\$$. 
O período de planejamento para depreciação das instalações foi de um ano, e para a depreciação de máquinas, implementos e equipamentos, o período foi coincidente com o número de meses estipulados para execução do projeto de investimento.

Os custos de oportunidade foram calculados considerando-se a taxa mínima de atratividade TMA. O custo de oportunidade do capital investido foi obtido em relação ao somatório das despesas operacionais (compra animal magro, controle sanitário, alimentação volumoso + concentrado, mão de obra contratada/diarista + assistência técnica e outras), para o período correspondente ao número de meses estipulados para cada nível de concentrado. Para o custo de oportunidade da terra, considerou-se a possibilidade de arrendamento anual da mesma pelo equivalente a $3 \%$ do valor do hectare para cultura. Determinou-se para cada animal o uso de 0,025 ha multiplicado pelo período correspondente ao número de meses estipulados para cada nível de concentrado.

O controle sanitário consistiu em aplicação de produto para controle de endo e ectoparasitas (ivermectina 1\%) e vacina contra febre aftosa, ambas em dosagem por animal conforme recomendações dos fabricantes.

O custo com alimentação foi obtido pelo produto entre o consumo total de volumoso e concentrado (em kg MS/animal), pelos seus respectivos custos/kg MS.

Outras despesas operacionais, como manutenção de instalações, máquinas, implementos e equipamentos, combustível, energia elétrica, frete, impostos e alimentação da mão de obra, foram estimadas pelo equivalente a $3,0 \%$ das despesas operacionais citadas anteriormente para estimar o custo de oportunidade do capital investido.

O custo operacional efetivo (COE) foi obtido pelo somatório das despesas operacionais: compra do animal magro, controle sanitário, alimentação com volumoso + concentrado, mão de obra contratada/diarista + assistência técnica e outras despesas operacionais. $\mathrm{O}$ custo operacional total (COT) representou o somatório do COE e depreciações. A margem bruta foi calculada como: receita com venda do animal gordo (R) - COE. A margem líquida foi obtida da seguinte maneira: R - COT. O lucro foi calculado como: R - (Fixos + Variáveis, ou COT + oportunidades). O custo/@ de ganho de peso foi estimado pela razão entre o custo total e o ganho de peso total $/ 30$.

Os indicadores financeiros foram estimados por animal, sendo: margem bruta (MB) em R\$; margem líquida (ML) em R\$; lucro em R\$; valor presente líquido (VPL) em $\mathrm{R} \$$; índice benefício: custo (IB:C) ou índice de lucratividade; retorno adicional sobre o investimento (ROIA) ou taxa de rentabilidade em \% a.m.; taxa interna de retorno (TIR) em \% a.m. e período de recuperação do investimento (payback) descontado (PBd) em meses (Souza e Clemente, 2009).

Com exceção de MB, ML e lucro, os demais indicadores não incluíram os custos de oportunidades na estimativa do custo total.

O delineamento experimental foi o inteiramente ao acaso, com quatro tratamentos e quatro repetições. Os dados foram submetidos à análise de variância, teste $\mathrm{F}$, análise de regressão linear polinomial, a 5\% de significância. O modelo matemático utilizado na análise de regressão polinomial foi o seguinte: $\hat{y}_{i}=b_{o}+b_{1} N_{i}+$ $\mathrm{b}_{2} \mathrm{NC}^{2}{ }_{\mathrm{i}}+\mathrm{e}_{\mathrm{i}}$, em que: $\hat{\mathrm{y}}_{\mathrm{i}}=$ variáveis dependentes estimadas, $b_{0}=$ intercepto, $b_{1}=$ coeficiente de regressão ou angular, $\mathrm{NC}_{\mathrm{i}}$ e $\mathrm{NC}_{\mathrm{i}}{ }=$ nível de concentrado na dieta (\% MS) de ordem linear e quadrática, respectivamente, $\mathrm{e}_{\mathrm{i}}=$ erro aleatório.

Como análise final, foi realizada a técnica multivariada de agrupamentos (cluster), visando avaliar possíveis agrupamentos de tratamentos em função dos indicadores VPL, IB:C, ROIA, TIR e PBd. Para isso, as variáveis citadas anteriormente foram padronizadas, sendo utilizada como medida de similaridade a distância euclidiana quadrática e a ligação de Ward. Detalhes metodológicos podem ser obtidos em Mingoti et al. (2007).

\section{RESULTADOS E DISCUSSÃO}

Verifica-se que os custos fixos estimados por animal (Tab. 5) apresentaram redução linear com o incremento no peso de abate. No entanto, acabaram contribuindo pouco em relação ao custo total (média de $0,72 \%$ ). 
Tabela 5. Médias para itens componentes dos custos e receitas estimados por animal, de acordo com o nível de concentrado na dieta

\begin{tabular}{|c|c|c|c|c|c|c|c|c|}
\hline \multirow{2}{*}{ Itens } & \multicolumn{4}{|c|}{ Nível de concentrado (NC), \% MS } & \multirow{2}{*}{ Média } & \multirow{2}{*}{$\begin{array}{l}\% \text { do } \\
\text { custo } \\
\text { total* }\end{array}$} & \multirow{2}{*}{$\mathrm{CV}, \%$} & \multirow{2}{*}{ Valor P } \\
\hline & 25 & 40 & 55 & 70 & & & & \\
\hline Custos fixos $(\mathrm{R} \$)^{1}$ & 13,33 & 12,25 & 12,25 & 10,15 & 12,00 & 0,72 & 3,43 & 0,0001 \\
\hline Custos variáveis $(\mathrm{R} \$)$ & 1660,51 & 1653,20 & 1665,61 & 1652,36 & $1.657,92$ & 99,28 & 4,65 & 0,9569 \\
\hline Compra do animal magro (R\$) & 1036,95 & 1021,00 & 1005,04 & 1028,44 & $1.022,86$ & 61,25 & 5,63 & 0,7866 \\
\hline Controle sanitário (R\$) & 4,36 & 4,36 & 4,36 & 4,36 & 4,36 & 0,26 & - & - \\
\hline Alimentação volumoso $(\mathrm{R} \$)^{2}$ & 234,13 & 184,01 & 139,59 & 84,91 & 160,66 & 9,62 & 5,16 & 0,0001 \\
\hline Alimentação concentrado $(\mathrm{R} \$)^{3}$ & 267,05 & 332,91 & 404,97 & 432,72 & 359,41 & 21,52 & 5,56 & 0,0001 \\
\hline Mão de obra contratada/diarista (R\$) & 9,96 & 8,98 & 8,98 & 7,67 & 8,90 & 0,53 & 3,33 & 0,5018 \\
\hline Assistência técnica $(\mathrm{R} \$)$ & 4,82 & 4,34 & 4,34 & 3,71 & 4,30 & 0,26 & 3,33 & 0,5014 \\
\hline Outros $(\mathrm{R} \$)$ & 46,72 & 46,67 & 47,02 & 46,85 & 46,82 & 2,80 & 4,63 & 0,8837 \\
\hline Oportunidade do capital investido ( $\mathrm{R} \$$ ) & 56,53 & 50,92 & 51,30 & 43,69 & 50,61 & 3,03 & 6,00 & 0,9178 \\
\hline Custo/ganho em @ (R\$/@) & 371,08 & 387,17 & 398,78 & 376,36 & 383,35 & - & 9,03 & 0,7755 \\
\hline Custo operacional efetivo (COE) $(\mathrm{R} \$)$ & 1603,98 & 1602,28 & 1614,31 & 1608,67 & $1.607,31$ & 96,25 & 4,63 & 0,8838 \\
\hline Custo operacional total (COT) $(\mathrm{R} \$)$ & 1614,59 & 1611,85 & 1623,89 & 1616,86 & $1.616,80$ & 96,82 & 4,61 & 0,9144 \\
\hline Custo total (fixos + variáveis) $(\mathrm{R} \$)$ & 1673,84 & 1665,45 & 1677,86 & 1662,51 & $1.669,92$ & - & 4,60 & 0,6856 \\
\hline Receita com venda do animal gordo $(\mathrm{R})(\mathrm{R} \$)$ & 1816,03 & 1761,66 & 1739,87 & 1804,53 & $1.780,52$ & - & 3,81 & 0,1529 \\
\hline
\end{tabular}

${ }^{1}$ (depreciação + oportunidade de instalações, máquinas, implementos e equipamentos + oportunidade da terra) $\hat{\mathrm{Y}}_{\mathrm{i}}=$ $14,97-0,063 * \mathrm{NC}_{\mathrm{i}}\left(\mathrm{R}^{2}=0,8538\right)$.

${ }^{2} \hat{\mathrm{Y}}_{\mathrm{i}}=316,27-3,278 * \mathrm{NC}_{\mathrm{i}}\left(\mathrm{R}^{2}=0,9809\right)$.

${ }^{3} \hat{\mathrm{Y}}_{\mathrm{i}}=179,54-3,789 * \mathrm{NC}_{\mathrm{i}}\left(\mathrm{R}^{2}=0,9078\right)$.

*Considerando a média de todos os níveis de concentrado em relação ao custo total. $\mathrm{R} \$=0,40 \mathrm{US} \$$.

A redução linear no custo com volumoso $(\mathrm{P}=0,0001)$ e o aumento linear no custo com concentrado $(\mathrm{P}=0,0001)$ não foram suficientes para resultar em diferenças no custo variável $(\mathrm{P}=0,9569)$, que representaram, em média, $99,28 \%$ do custo total ( $\$$ \$ 1.657,92), considerando que os itens relacionados com a alimentação foram os mais representativos.

Ao avaliarem diferentes níveis de concentrado na dieta de novilhos confinados, Pacheco et al. (2014a) verificaram que, com o incremento no teor de concentrado na dieta de $40 \%$ (menor nível) para $80 \%$ (maior nível), o custo com volumoso (cana-de-açúcar in natura picada) reduziu $67 \%$, enquanto o custo com concentrado aumentou $122 \%$, ambos de maneira significativa, resultando em incremento linear nos custos variáveis.

Verifica-se que as características relacionadas com o desempenho (Tab. 3) e o custo da dieta (Tab. 4) não resultaram em alterações no custo total, bem como na receita com a venda dos animais gordos (Tab. 5). Provavelmente a redução no tempo de alimentação dos animais com o aumento no nível de concentrado tenha contribuído parcialmente para isto, o que, combinado com o comportamento quadrático no consumo da dieta (Tab. 3), refletiu na similaridade dos custos variáveis. Diferenças entre os níveis de 40,60 e 80\% de concentrado na dieta de novilhos zebuínos foram verificadas no estudo de Pacheco et al. (2014a), com incremento linear de R\$2,22/animal no custo total a cada um ponto percentual de aumento no nível de concentrado, no entanto a receita foi similar entre os tratamentos.

Em relação à participação relativa dos itens componentes do custo total, os principais foram ranqueados da seguinte maneira, em ordem decrescente: compra do animal magro, alimentação concentrado e alimentação volumoso. Lopes e Magalhães (2005), Pacheco et al. (2006), Missio et al. (2009), Pacheco et al. (2012) e Pacheco et al. (2014a) verificaram resultados concordantes com o presente estudo, o que indica relevância prioritária quanto às estratégias de comercialização e sugere o monitoramento de cotações ao longo do(s) ano(s), que podem, por exemplo, auxiliar na tomada de decisão desse sistema de terminação (Pacheco et al., 2014a). 
$\mathrm{Na}$ análise conjunta dos indicadores financeiros (Tab. 6), verifica-se similaridade $(P>0,05)$ entre os níveis de concentrado, no entanto com viabilidade econômica.

Simões et al. (2006) esclarecem sobre a interpretação dos indicadores $\mathrm{MB}, \mathrm{ML}$ e lucro como sendo indicativos da possibilidade de viabilidade econômica no curto, médio e longo prazos, respectivamente. No curto prazo (MB), é considerada a diferença entre a receita $e$ as despesas operacionais, o que se torna muito relevante em investimentos como a terminação em confinamento, pois o ciclo de cada lote de animais (entrada quando magro até a saída quando gordo) é menor do que um ano. No médio prazo (ML), é considerada a diferença entre a receita e as despesas operacionais + depreciações, sendo também importante, pois as depreciações relacionadas com instalações, máquinas e implementos têm vida útil de alguns anos. No longo prazo (lucro), é considerada a diferença entre a receita e as despesas operacionais + depreciações + oportunidades, que adicionalmente considera o valor do dinheiro no tempo.

Tabela 6. Indicadores financeiros de acordo com o nível de concentrado na dieta, estimados por animal e apenas durante o período de confinamento

\begin{tabular}{lrrrrrrrr}
\multirow{2}{*}{\multicolumn{1}{c}{ Indicadores }} & \multicolumn{3}{c}{ Nível de concentrado, \% MS } & Média & CV, & Valor P \\
\cline { 2 - 6 } & 25 & 40 & 55 & 70 & & $\%$ & \\
\hline Margem bruta (R - COE), R\$ & 212,05 & 159,38 & 125,56 & 195,86 & 173,21 & 17,59 & 0,2529 \\
Margem líquida (R - COT), R\$ & 201,43 & 149,81 & 115,99 & 187,68 & 163,73 & 18,69 & 0,2529 \\
Lucro (R - custo total), R\$ & 142,19 & 96,22 & 62,01 & 142,03 & 110,61 & 29,42 & 0,2327 \\
Valor presente líquido - VPL, R\$ & 126,38 & 90,89 & 43,18 & 112,80 & 93,31 & 35,13 & 0,2505 \\
Índice benefício: custo - IB: C & 1,065 & 1,049 & 1,028 & 1,049 & 1,048 & 1,36 & 0,2844 \\
Retorno adicional sobre investimento - ROIA, \% a.m. & 1,60 & 1,20 & 0,67 & 1,20 & 1,17 & 29,99 & 0,2844 \\
Taxa interna de retorno - TIR, \% a.m. & 2,47 & 2,03 & 1,40 & 2,27 & 2,04 & 20,19 & 0,2364 \\
Payback descontado - PBd, meses & 3,76 & 3,81 & 3,89 & 3,81 & 3,82 & 1,36 & 0,2745 \\
\hline
\end{tabular}

$\mathrm{R} \$=0,40$ US\$.

No estudo de Pacheco et al. (2014a), em que avaliaram diferentes níveis de concentrado na dieta de novilhos zebuínos abatidos com $450 \mathrm{~kg}$, não houve diferenças significativas para $\mathrm{MB}$, ML e lucro, sendo todas as médias negativas. Pacheco et al. (2012) avaliaram os pesos de abate de 425, 467 e $510 \mathrm{~kg}$ de novilhos mestiços Charolês $\mathrm{x}$ Nelore em relação à viabilidade econômica e verificaram similaridade para os indicadores $\mathrm{MB}$, ML e lucro $(\mathrm{P}<0,05)$, sendo apenas os dois primeiros com médias positivas. No estudo de Pacheco et al. (2014b), em que trabalharam com novilhos Charolês, os indicadores financeiros foram negativos para todos os pesos de abate $(421,461$ e $495 \mathrm{~kg}$ ), enquanto em Pacheco et al. (2015), em que analisaram a viabilidade econômica da terminação de novilhos Red Angus abatidos com $340,373,396$ e $430 \mathrm{~kg}$, o peso de abate influenciou MB e ML, com comportamento quadrático conforme análise de regressão (valores positivos). Ressalta-se que os três últimos estudos citados utilizaram cotações históricas de nove anos consecutivos (2004 a 2012).
$\mathrm{Na}$ avaliação do efeito do incremento no nível de concentrado na dieta $(22,40,59$ e $79 \%)$ de tourinhos confinados dos 14 aos 16 meses de idade, Missio et al. (2009) verificaram diminuição linear da renda líquida e da lucratividade com o aumento do concentrado na dieta ao simularem preços do custo com concentrado em percentagem do preço do $\mathrm{kg}$ do boi gordo. Conforme os autores, nos maiores níveis de concentrado, a terminação dos animais foi inviabilizada economicamente quando o preço do concentrado atingiu cerca de $26 \%$ do preço do boi gordo, e quando o preço do concentrado representou $53 \%$ do valor do preço do boi gordo, todos os níveis de concentrado utilizados se tornaram economicamente inviáveis.

Dos indicadores financeiros mais difundidos, o valor presente líquido - VPL - é o método de análise de investimento mais conhecido e utilizado (Souza e Clemente, 2009). Verifica-se, na Tab. 6, que houve similaridade para VPL entre os diferentes níveis de concentrado, resultado importante para tomada de decisão em 
relação aos investimentos em confinamento de bovinos como atividade principal, ou seja, desconsiderando seus benefícios indiretos dentro de um sistema de produção de bovinos de corte. Isto porque as atividades/fatores/manejo envolvidos com a dieta sempre são preocupantes no gerenciamento desse negócio, tendo em vista sua relação estreita com a viabilidade econômica. Nem sempre elevar o nível de concentrado será a melhor estratégia, mesmo considerando o mesmo ano produtivo, em razão das oscilações nas cotações nos itens componentes dos custos da dieta.

Conforme Souza e Clemente (2009), o VPL representa o quanto se recupera do investimento inicial, considerando sua remuneração quando aplicado na taxa mínima de atratividade - TMA. Dessa maneira, a média de R \$93,31/animal para VPL (Tab. 6) significa o excedente após o projeto recuperar o investimento inicial e remunerar aquilo que teria sido ganho se o capital para esse investimento tivesse sido aplicado na TMA. a qual, no presente estudo, usada como referência foi a Selic.

No estudo de Pacheco et al. (2014a), os quais avaliaram o uso de 40, 60 ou $80 \%$ de concentrado na dieta de novilhos confinados por 90 dias, houve similaridade para VPL e com valores negativos, indicando inviabilidade da atividade. Demais pesquisas que utilizaram a metodologia semelhante à do presente estudo (Resende Filho et al., 2001; Pacheco et al., 2012; Pacheco et al., 2015) estimaram VPL positivo para os diferentes pesos de abate considerados.

Quanto ao índice benefício:custo - IB:C, este representa uma medida do quanto se espera ganhar por unidade de capital investido (Souza e Clemente, 2009), ou seja, o quanto se espera de retorno de cada R\$ 1,00 investido. Quando o fluxo de entradas descontado é maior do que o fluxo de saídas descontado, o projeto apresenta viabilidade e merece continuar sendo analisado.

No presente estudo, o IB:C médio foi de 1,048, ou seja, a cada $\mathrm{R} \$ 1,00$ mobilizado no projeto de investimento, esperava-se retirar, após o horizonte de planejamento dele (quatro meses para o presente estudo - Tab. 2), R\$ 1,048 após isento o ganho que se teria caso esse $R \$ 1,00$ tivesse sido aplicado na TMA, conforme Souza e Clemente (2009).
Similaridade para IB:C foi relatada no estudo de Pacheco et al. (2014a) ao avaliarem diferentes níveis de concentrado, no entanto a média foi de 0,8994. Valores abaixo de 1,00 para IB:C foram estimados nos estudos de Mello et al. (2009) e Pacheco et al. (2014b) ao estudarem diferentes pesos de abate, enquanto em Pacheco et al. (2012) e Pacheco et al. (2015) os valores foram positivos.

Quando o horizonte de planejamento do projeto de investimento não coincide com o período definido para a TMA (quatro meses vs. um mês, respectivamente, para o presente estudo), Souza e Clemente (2009) afirmam que a rentabilidade estimada pelo IB:C não pode ser comparada imediatamente com a TMA. É necessário definir uma taxa equivalente para o mesmo período da TMA, sendo esta representativa da rentabilidade esperada do projeto para o mesmo período da taxa de atratividade, denominada retorno adicional sobre o investimento - ROIA.

Verifica-se, na Tab. 6, estimativa média de $1,17 \%$ a.m. para ROIA, similar $(\mathrm{P}>0,05)$ para os diferentes níveis de concentrado na dieta. Esse valor representa a rentabilidade do projeto além da TMA $(0,8667 \%$ a.m. ou $10,91 \%$ a.a. no presente estudo).

Conforme estimativas de Pacheco et al. (2014a), não houve diferença significativa para ROIA ao avaliarem três níveis de concentrado na dieta de novilhos confinados, com média negativa de $10,06 \%$ a.m.. No entanto, quando analisaram diferentes pesos de abate, Pacheco et al. (2012) e Pacheco et al. (2015) verificaram valores positivos de até $12 \%$ a.m.

Em relação à taxa interna de retorno - TIR, Souza e Clemente (2009) comentam que tanto pode ser usada para analisar a dimensão retorno como a dimensão risco. Na primeira, ela pode ser interpretada como um limite superior para a rentabilidade de um projeto de investimento. Essa informação é relevante se, para o projeto em análise, não se souber qual o valor da TMA. Pela dimensão risco, a informação da TIR é mais relevante e pode ser interpretada como um limite superior para a variabilidade da TMA. Isto decorre do fato de o VPL ir decrescendo à medida que a TMA se aproxima da TIR. 
No presente estudo, a TIR foi similar entre os diferentes níveis de concentrado $(\mathrm{P}>0,05)$, com média de 2,04\% a.m. O fato de esse valor ser 2,3 vezes superior à TMA $(0,87 \%$ a.m. $)$ significa que o projeto apresenta baixo risco em relação ao seu retorno financeiro, conforme esclarecimentos de Souza e Clemente (2009). No estudo de Pacheco et al. (2014a), houve similaridade entre os níveis de concentrado para TIR, com média de $-3,84 \%$ a.m. (TMA $=0,4868 \%$ a.m.). Demais estudos que avaliaram diferentes pesos de abate reportam valores de TIR positivos (Fernandes et al., 2007; Coan et al., 2008; Pacheco et al., 2012; Pacheco et al., 2015).

Para o último indicador financeiro apresentado na Tab. 6, payback descontado - PBd, verificamse valores muito próximos entre os níveis de concentrado avaliados $(\mathrm{P}>0,05)$, com média de 3,82 meses. Esse valor representa o número de períodos necessários para que o fluxo de benefícios supere o capital investido. Para Souza e Clemente (2009), o PBd pode ser considerado outro indicador de risco de projetos de investimento, dessa maneira, quanto mais próximo o valor do PBd for do final do horizonte de planejamento, maior é o risco. Pacheco et al. (2014a) comentam que, na análise econômica da terminação em confinamento de bovinos, esperase que os valores de PBd sejam muito próximos do número de períodos para execução do projeto de investimento, pois a única ou principal receita é oriunda da venda dos animais gordos, sendo esta coincidente com o último período.

Ao se observarem os resultados da Tab. 6, argumenta-se que a análise conjunta de diversos indicadores financeiros proporciona melhores condições para tomada de decisão, pois atende as mais diversas preferências por parte dos investidores. No entanto, ao mesmo tempo, sugere o agrupamento desses diversos indicadores na possibilidade de avaliar $o$ comportamento entre os diferentes níveis de concentrado, com a possibilidade, inclusive, de apresentar resultados discordantes entre as análises multivariada e univariada.

Na Fig. 1, consta o dendograma da análise de cluster, considerando seis indicadores financeiros em conjunto e os níveis de concentrado comparados e agrupados conforme grau de similaridade. Foram considerados três grupos, sendo o nível de concentrado de $55 \%$ o que apresentou maior discrepância em relação aos demais níveis (similaridade de -2,57), enquanto $25 \%$ obtiveram nível de similaridade de 90,16 em relação a 40 e $70 \%$. Esses dois últimos níveis de concentrado, por sua vez, foram os mais próximos (nível de similaridade de 96,02).

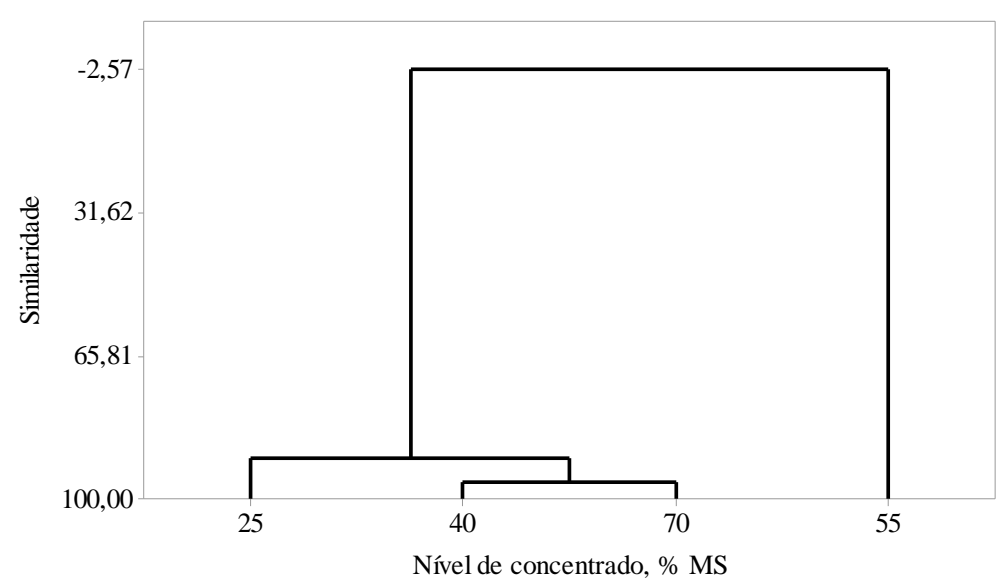

Figura 1. Dendograma obtido da análise de cluster dos níveis de concentrado, considerando-se diversos indicadores financeiros (VPL, IB:C, ROIA, TIR, PBd).

Em suas reflexões sobre análises univariada e multivariada, Saccenti et al. (2014) revisaram estudos que mostraram que os resultados de análises uni e multivariada não necessariamente coincidem. Esses autores discorrem sobre as razões de esses resultados não terem de ser os mesmos e sugerem, ainda, a utilização de ambos os resultados porque os métodos realmente 
mostram questões diferentes. Eles também aconselham não procurar validação de resultados da análise univariada por meio da análise multivariada e vice-versa. Isto significa que os dois métodos podem proporcionar resultados complementares, mas esses resultados devem ser interpretados dentro do quadro estatístico (uni ou multivariada) com o qual tenham sido produzidos.

\section{CONCLUSÕES}

Os indicadores financeiros demonstraram viabilidade econômica da terminação em confinamento de novilhos Aberdeen Angus, independentemente do nível de concentrado. Os resultados foram coincidentes entre os indicadores financeiros avaliados, permitindo maior base criteriosa para a tomada de decisão.

\section{REFERÊNCIAS}

ANUÁRIO DA PECUÁRIA BRASILEIRA. São Paulo: Instituto FNP, 2015. 280p.

COAN, R.M.; REIS, R.A.; RESENDE, F.D. et al. Viabilidade econômica, desempenho $\mathrm{e}$ características de carcaça de garrotes em confinamento alimentados com dietas contendo silagem de capins tanzânia ou marandu ou silagem de milho. Rev. Bras. Zootec., v.37. p.311-318, 2008.

COSTA JUNIOR, C.; GOULART, R.S.; ALBERTINI, T.Z. et al. Brazilian beef cattle feedlot manure management: a country survey. $J$. Anim. Sci., v.91, p.1811-1818, 2013.

FERNANDES, A.R.M.; SAMPAIO, A.A.M.; HENRIQUE, W. et al. Avaliação econômica e desempenho de machos e fêmeas Canchim em confinamento alimentados com dietas à base de silagem de milho e concentrado ou cana-deaçúcar e concentrado contendo grãos de girassol. Rev. Bras. Zootec., v.36, p.855-864, 2007.

LOPES, M.A.; MAGALHÃES, G.P. Análise da rentabilidade da terminação de bovinos de corte em condições de confinamento: um estudo de caso. Arq. Bras. Med. Vet. Zootec., v.57, p.374379, 2005.
MELLO, R.; RESENDE, F.D.; QUEIROZ, A.C. et al. Bio-economicity of the finishing phase on feedlot of crossbred young bulls slaughtered at different body weights. Rev. Bras. Zootec., v.38, p.109-121, 2009.

MILLEN, D.D.; PACHECO, R.D.L.; ARRIGONI, M.D.B. et al. A snapshot of management practices and nutritional recommendations used by feedlot nutritionists in Brazil. J. Anim. Sci., v.87, p.3427-3439, 2009.

MINGOTI, S.A. Análise de dados através de métodos de estadística multivariada. Belo Horizonte: UFMG, 2007. 295p.

MISSIO, R.L.; BRONDANI, I.L.; FREITAS, L.S. et al. Desempenho e avaliação econômica da terminação de tourinhos em confinamento alimentados com diferentes níveis de concentrado na dieta. Rev. Bras. Zootec., v.38, p.1309-1316, 2009.

NUTRIENTS requeriments of beef cattle. 7.ed. Washington: National Academy of Science 1996. $244 p$.

PACHECO, P.S.; RESTLE, J.; VAZ, F.N. et al. Avaliação econômica da terminação em confinamento de novilhos jovens e superjovens de diferentes grupos genéticos. Rev. Bras. Zootec., v.35, p.309-320, 2006.

PACHECO, P.S.; RESTLE, J.; VAZ, F.N. et al. Viabilidade econômica da terminação em confinamento de novilhos abatidos com diferentes pesos. Pesqui. Agropecu. Gaúcha, v.18, p.135-145, 2012.

PACHECO, P.S.; RESTLE, J.; VALENÇA, K.G. et al. Análise econômica determinística da terminação em confinamento de novilhos abatidos com distintos pesos. Cienc. Anim. Bras., v.15, p.420-427, 2014b.

PACHECO, P.S.; SILVA, R.M.; PADUA, J.T. et al. Análise econômica da terminação de novilhos em confinamento recebendo diferentes proporções de cana-de-açúcar e concentrado. Semin. Cienc. Agrár., v.35, p.999-1012, 2014a.

PACHECO, P.S.; VAZ, F.N.; RESTLE, J. et al. Deterministic economic analysis of feedlot Red Angus young steers: slaughter weights and bônus. Cienc. Rural, vol.45, 2015. 
RESENDE FILHO, M.A.; BRAGA, M.J.; RODRIGUES, R.V. Sistemas de terminação em confinamento: perspectivas para dinamização da cadeia produtiva da carne bovina em Minas Gerais. Rev. Bras. Econ., v.55, p.107-131, 2001.

ROSA, J.R.P. Exigências energéticas e proteicas de novilhos de Aberdeen Angus submetidos ou não ao ganho compensatório. 2006. 149f. Tese (Doutorado em Zootecnia) - Faculdade de Agronomia, Universidade Federal do Rio Grande do Sul, Porto Alegre, RS.
SACCENTI, E.; HOEFSLOOT, H.C.J.; SMILDE, A.K. et al. Reflections on univariate and multivariate analysis of metabolomics data. Metabolomics, v.10, p.361-374, 2014.

SIMÕES, A.R.P.; MOURA, A.D.; ROCHA, D.T. Avaliação econômica comparativa de sistemas de produção de gado de corte sob condições de risco no Mato Grosso do Sul. Rev. Econom. Agroneg., v.5, p.51-72, 2006.

SOUZA, A.; CLEMENTE, A. Decisões financeiras e análise de investimentos. 6.ed. São Paulo: Atlas, 2009. 186p. 